
\title{
3 Research Square \\ Traditional Chinese medicine for ischemic heart disease: clinical manifestations and objective indicators
}

Jiangquan Liao

China-Japan Friendship Hospital https://orcid.org/0000-0002-8445-0433

Jiaxing Tian

Guang'anmen hospital

Mingjing Shao

China-Japan Friendship Hospital

Zhe Wang

Beijing University of Chinese Medicine

Kangkang Wei

Beijing University of Chinese Medicine

Jiangmeng Chang

Beijing University of Chinese Medicine

Xiaoqiong Zhang

Beijing University of Chinese Medicine

Ming Chen

Beijing University of Chinese Medicine

Xianlun Li ( $\square$ leexianlun@163.com)

China-Japan Friendship hospital

Jinhang Du ( $\sim$ du7135@sina.com)

China-Japan Friendship Hospital

\section{Research}

Keywords: Traditional Chinese medicine, ischemic heart disease, clinical manifestations, objective indicators, meta-analysis

Posted Date: April 1st, 2020

DOI: https://doi.org/10.21203/rs.3.rs-18920/v1

License: (a) (i) This work is licensed under a Creative Commons Attribution 4.0 International License.

Read Full License 


\section{Abstract}

Background: The clinical practice of Traditional Chinese medicine (TCM) has a history of more than 2000 years. Modern clinical trials and experimental researches of TCM have been conducted for decades and provided support for the application of TCM in the prevention and treatment of ischemic heart disease (IHD). However the level of evidence and the proper application of TCM were still barely satisfactory.

Methods: In this study, we divided IHD into 5 different stages, including stable angina, unstable angina, acute myocardial infarction, post myocardial infarction and chronic heart failure. Then we systematically reviewed and meta-analyzed the existing RCTs on both clinical manifestations and objective indicators, in these 5 aspects.

Results: The results indicate that TCM can both improve the clinical manifestations and ameliorate the objective parameters in different courses of IHD. Some of the improvements lead to potential long-term benefits.

Conclusions: TCM is effective on CVD in different stages of diseases, both in improving clinical manifestations and objective indicators. To acquire more solid and comprehensive evidence of TCM in treating CVD, more rigorously designed RCTs with longer follow-up duration are warranted.

\section{Background}

Cardiovascular diseases (CVD) have been recognized as the leading cause of death and long term disability across the world for years ${ }^{1}$. According to the Global Burden of Death (GBD) ${ }^{2,3}$ Ischemic Heart Disease (IHD) is the number 1 cause of deaths in cardiovascular diseases both in male and female. IHD has caused 116.88 deaths per million populations in 2017, which has increased $7.51 \%$ from $1990^{4}$. Current circumstance of CVD, especially of IHD, is far from satisfying. Traditional Chinese medicine (TCM) has shown its possible role in the prevention and management of IHD. Numerous researches were held within the framework of modern medicine, some of which have solid sound of the efficacy of TCM ${ }^{5-}$ 7 .

TCM has a history of more than 2000 years. During its developing process, TCM formed a self-consistent theoretical system. The most fundamental elements of TCM are holism and syndrome differentiation ${ }^{8}$. Under the guidance of TCM theory, experienced clinicians can prescribe specific formula or other therapy for different individuals and achieve efficacy in certain aspects. Since the theory of TCM is formed and gradually developing along years of practice, the understanding and utilization of TCM may differ in different clinicians. It gives TCM variety and vitality, which also makes TCM difficult to embrace and extend worldwide as lacking of objective, quantitative and consistent evaluation criteria.

As we mentioned above, TCM has always been developing, which was determined by its experienceguiding nature. New generation TCM clinicians have used modern medical equipment to enhance their information acquisition, and established more objective diseases diagnosis and syndrome differentiation 
criteria. Scientific researches of TCM were launched to elucidate the effectiveness and mechanisms of TCM. TCM, or integrative medicine has help preventing and treating diseases across the world, not just in east of Asia? .

Under this circumstance, it is worth to gather the evidence of TCM treating CVD, to provide faith in treating CVD with TCM as primary or alternative therapy, and help decreasing the mortality and morbidity of CVD worldwide. Here, we aim to evaluate the efficacy and potential advantages of TCM in different course of IHD. We searched for published studies of RCTs with relatively fine quality, and systematically assessed the efficacy and safety of TCM therapy for the major clinical stages of IHD, including stable angina (SA), unstable angina (UA), acute myocardial infarction (AMI), post myocardial infarction (PMI) and chronic heart failure (CHF) caused by IHD.

\section{Methods}

\section{Search strategy and inclusion/exclusion criteria}

Since English and Chinese are the major publication language that TCM related researches used ${ }^{10}$, electronic databases including Medline, EMBASE, the Cochrane Library, the China National Knowledge Internet, the China biology medicine, Wanfang, and VIP databases were searched up to 1 December 2018 for all studies evaluating the effect of TCM on SA, UA, AMI, PMI and CHF. The key words used in the search were 'traditional Chinese', or 'traditional Chinese medicine', or 'Chinese medicine' in combination with each of the following terms: 'coronary artery', 'coronary heart', 'angina', 'ischemia', 'ischemic', 'myocardial infarction', 'percutaneous coronary intervention', 'coronary artery bypass graft', 'revascularization' or 'heart failure'. The specific search algorithms were adjusted for each database. The authors of the articles were contacted for detailed information if necessary.

The studies we included should meet following criteria: 1) Study participants were diagnosed as SA, UA, $\mathrm{Ml}$, or $\mathrm{CHF}$, did or did not underwent percutaneous coronary intervention $(\mathrm{PCl})$, coronary artery bypass grafting ( $C A B G)$ or thrombolysis. 2) Study should be randomized clinical trial, which compared the efficacy and quantitative parameters of TCM medication with placebo or contemporary medication. 3 ) Follow-up in each study should $\geq 4$ weeks. 4) Study quality was considerably fine, with a Jadad score ${ }^{11} \geq 2$. We excluded studies with the following features: 1 ) Studies were nonrandomized or without description of randomization method. 2) Studies without definite diagnostic criteria. 3) Studies compared different TCM medications. When 2 articles reported the results from the same research, the articles with more data was included.

We have searched and investigated other systematic reviews of TCM, most of which would exclude the studies without objective laboratory measurements or physical parameters. One of the unique characteristics of TCM is emphasizing the symptoms of diseases. The cluster and pattern of symptoms can be both the evaluation of the features of disease, and the efficacy of the TCM medication. And by the Guiding Principles for Clinical Research of New Chinese Medicines, which was drafted and published by 
State Drug Administration of China, most of TCM related studies evaluated the clinical efficacy with semiquantitative methods and unified standard. Hence we included those studies which used clinical efficacy evaluated with well-adapted standard as primary parameters.

\section{Statistical analysis}

Data were analyzed using Review Manager v5.3 $3^{12}$ and Microsoft excel 2016. Meta-analysis was conducted if the included studies were no less than 2. For dichotomous outcome the pooled relative risk (RR) with 95\% confidence interval (Cl) was used as the effect measure. For the continuous outcome, standard mean difference (SMD) was used as the effect measure. The model used to pool the data was Random effect model, since the intervention between studies were different.

\section{Results}

\section{General information}

A flow diagram of the literature search and study selection is shown in Figure 1. We included 77 eligible studies, in which 11 were in English, 66 were in Chinese. The publication date ranged from 2006 to 2018. The participant amount in single group varied from 20 to 2441, the average amount was 79 (the average amount was 49 if one of the largest scale RCT was ruled out). A total of 12544 subjects were included in this research. The quality of the studies were considered mostly moderate, 6 of them had a Jadad score of 5,12 of them had a Jadad score of 3 to 4 , and the rest of them (60 studies) had a Jadad score of 2. Generally, there were 25 studies of SA, 18 studies of UA, 12 studies of AMI, 7 studies of PMI, and 16 studies of CHF. The detail information of included studies were listed in Table 1.

\section{TCM for Stable Angina}

25 RCTs (No. 1-25 in Table 1) evaluated TCM treating SA were included and assessed. The sample size ranged from 46 to 239 participants, with average of 88.68. The intervention duration ranged from 4 to 12 weeks, with average of 6.18 weeks. The methodological quality of the included RCTs was generally low. 2 of 25 RCTs had a Jadad score of 5, and 2 had a Jadad score of 4, 2 had score of 3 and 19 had score of 2. The major and secondary outcomes we included and assessed were clinical efficacy, angina efficacy, angina frequency, nitrates consumption, ECG efficacy, and levels of total cholesterol (TC), low density lipoprotein-cholesterol (LDL-C), high density lipoprotein-cholesterol (HDL-C), triglyceride (TG).

SA is now considered stable ischemic heart disease (SIHD), a stage of coronary heart disease (CHD) with certain manifestations of cardiac ischemia, the existence of cardiovascular risk factors, and the potential risk of developing into acute coronary syndromes (ACS). According to the latest guideline, the management of SA is comprised of the management of risk factors (i.e. hypertension, dyslipidemia, diabetes mellitus) and the control of ischemic symptoms ${ }^{13}$. The control of angina pectoris is important in the management of SA, especially to those patients without any risk factor or had taken action on the risk factors. The reduction of angina frequency, degree of pain or discomfort, consumption of nitrates 
compose of the clinical efficacy of SA treatment. Hence we conducted meta-analysis of clinical efficacy, angina efficacy, angina frequency and nitrates consumption in TCM treating SA.

In the meta-analysis of clinical efficacy, 20 RCTs were included, and had shown significant difference of RR $(1.25,95 \% \mathrm{Cl}, 1.17$ to 1.34$)$. Statistical heterogeneity in this model was considered low $(P=0.22$, $\mathrm{I}^{2}=19 \%$ ) (Figure 2A). TCM had a significant effect in improving clinical efficacy. 11 RCTs were included in the meta-analysis of angina efficacy, and had shown significant difference of $\mathrm{RR}(1.23,95 \% \mathrm{Cl}, 1.11$ to 1.37). Statistical heterogeneity in this model was considered high $\left(P=0.005, I^{2}=60 \%\right)$ (Figure 2B). 6 RCTs were included in the meta-analysis of angina frequency, the RR was $-0.82(95 \% \mathrm{Cl},-1.25$ to -0.39$)$ (Figure 2C). 5 RCTs were included in the meta-analysis of nitrate consumption, the RR was $-1.13(95 \% \mathrm{Cl},-1.68$ to -0.59) (Figure 2D). These analyses indicated TCM could both ameliorate the manifestation of angina and reduce the angina frequency and consumption of nitrates, together improved the clinical efficacy of SA. 13 RCTs also paid close attention to the ECG efficacy. Meta-analysis showed significant improvement of TCM in ECG, with RR of 1.23 and $95 \% \mathrm{Cl} 1.14$ to 1.33 (Figure 2E). ECG efficacy indicated that TCM can improve stable angina in the aspect of ECG.

Analyses also showed that TCM can improve dyslipidemia in SA. 9 RCTs reported TC level after intervention. Meta-analysis showed that TCM can significantly lower TC in SA (SMD -1.22, 95\% Cl -1.58 to -0.87 ) (Figure 3A). 10 RCTs showed that TCM can significantly lower LDL-C in SA $(-0.79,95 \% \mathrm{Cl}-1.06$ to -0.53 ) (Figure 3B). TCM can also lower TG in meta-analysis of 9 RCTs $(-0.86,95 \% \mathrm{Cl}-1.18$ to -0.21$)$ (Figure 3D). As for the protective cholesterol, TCM can increase the level of HDL-C, supported by the metaanalysis of $8 \mathrm{RCTs}(0.6,95 \% \mathrm{Cl} 0.27$ to 0.94$)$ (Figure $3 \mathrm{C}$ ).

Dyslipidemia is one of the major risk factors of cardiovascular disease, and the levels of cholesterol influence the stratification of the CVD risk categories, hence affect the expectation of cardiovascular event and the treatment intensity ${ }^{14}$. These meta-analyses indicated that TCM can adjust the levels of cholesterol, and serve as effective therapy for SA in both clinical manifestation and risk factors.

\section{TCM for Unstable Angina}

18 RCTs (No. 26-43) evaluated TCM treating UA were included and assessed. The sample size ranged from 60 to 244 participants, with average of 103.17. The intervention duration ranged from 4 to 12 weeks, with average of 4.78 weeks. The methodological quality of the included RCTs was generally low. Only 1 of 18 RCTs had a Jadad score of 4 and 3 , and the rest of them had score of 2 . The major and secondary outcomes we included and assessed were clinical efficacy, angina efficacy, angina frequency, nitrates consumption, ECG efficacy, levels of LDL-C and C-reactive protein (CRP). 1 of 18 RCTs compared TCM with placebo, and the rest of them compared TCM with blank intervention, on the basis of conventional therapy, including antiplatelet, beta blocker, anticoagulant, statins, ACEI/ARB, long- or short-active nitrates, and hypotensor, hypoglycemics if necessary.

14 of 18 RCTs had reported the clinical efficacy after intervention. Meta-analysis had shown significant difference of $\operatorname{RR}(1.20,95 \% \mathrm{Cl}, 1.12$ to 1.27$)$. Statistical heterogeneity in this model was considered 
moderate to high ( $P=0.04, I^{2}=43 \%$ ) (Figure $4 A$ ). As for the angina efficacy, 6 RCTs were included in the meta-analysis and had shown significant difference of $\mathrm{RR}(1.25,95 \% \mathrm{Cl}, 1.14$ to 1.36$)$. Statistical heterogeneity in this model was considered low $\left(P=0.89, \mathrm{I}^{2}=0 \%\right)$ (Figure 4B). The RCTs documented angina frequency and nitrates consumption were relatively fewer (2 RCTs in each comparison). Metaanalysis were conducted in these 2 comparison, and had shown significant difference in RR (angina frequency: $-1.95,95 \% \mathrm{Cl}-2.41$ to -1.49 , heterogeneity $\mathrm{P}=0.14, \mathrm{I}^{2}=54 \%$; nitrates consumption: $-2.12,95 \% \mathrm{Cl}$ -3.02 to -1.22 , heterogeneity $P=0.004, I^{2}=88 \%$ ) (Figure $4 C$ and D). These analyses indicated TCM could improve the clinical efficacy of SA, probably by ameliorate the manifestation of angina and reduce the angina frequency and consumption of nitrates.

7 RCTs had documented the ECG efficacy after intervention. Meta-analysis showed significant improvement of TCM in ECG, with RR of 1.25 and $95 \% \mathrm{Cl} 1.14$ to 1.36 . Statistical heterogeneity in this model was considered low $\left(P=0.89, I^{2}=0 \%\right.$ ) (Figure $4 E$ ). ECG efficacy indicated that TCM can improve unstable angina in the aspect of ECG.

As for the laboratory parameters, LDL-C and CRP were synthetize respectively. Meta-analysis shown significant difference in CRP $(-1.10,95 \% \mathrm{Cl}-1.64$ to -0.55 , with Random effect model, heterogeneity $\mathrm{P}<0.00001, \mathrm{I}^{2}=90 \%$ ) (Figure $5 \mathrm{~A}$ ) but not in LDL-C (0.12, $95 \% \mathrm{Cl}-0.75$ to 0.99$)$ (Figure $5 \mathrm{~B}$ ). Meta-analyses of 2 out of 3 RCTs that LDL-C included showed no significant difference either. Data of other conventional cholesterol was not sufficient enough for systematic review in these 18 RCTs concerning UA. The efficacy of TCM for CVD risk factors in UA needs more evidence to be proved.

\section{TCM for AMI}

11 RCTs (No. 44-54) evaluated TCM treating AMI were included and assessed. All the participants in every RCTs were diagnosed myocardial infarction with formally published criteria. The intervention time nodes were around the AMI and coronary recanalization. Respectively, 2 RCTs were 30 minutes to 2 days before recanalization; 1 RCTs were 12 to 55 days after recanalization; 8 RCTs were immediately after recanalization. The sample size ranged from 40 to 219 participants, with average of 111.18. The intervention duration ranged from 4 to 24 weeks, with average of 7.82 weeks. The methodological quality of the included RCTs was moderate. 2 of 11 RCTs had a Jadad score of 5 , and 1 had a Jadad score of 4 , 2 had score of 3 and 6 had score of 2. The major and secondary outcomes we included and assessed were clinical efficacy, major adverse cardiovascular events (MACE), cardiovascular death (CD) and left ventricular ejection fraction (LVEF), according to the data in RCTs we included.

7 of 11 RCTs had reported the clinical efficacy after intervention. All of them compared TCM with blank intervention, with the basis of antiplatelet, anticoagulant, beta blocker, ACEI. Participants in 1 of the RCTs underwent thrombolysis, and in another RCT, participants underwent PCI. The rest 5 RCTs used conservative treatment as described above. Meta-analysis had shown significant difference of RR (1.11, $95 \% \mathrm{Cl}, 1.05$ to 1.18$)$. Statistical heterogeneity in this model was considered low $\left(P=0.36, I^{2}=11 \%\right.$ ) (Figure 6). This result indicated that TCM can improve the clinical efficacy of AMI. 
The average follow-up duration of these RCTs were 7.82 weeks ( 4 to 24 weeks), hence the short-term outcomes would be the major concern of TCM intervention in these RCTs. 4 of the RCTs had documented incidence of MACE in each group after intervention. Meta-analysis showed significant difference of RR $(0.57,95 \% \mathrm{Cl} 0.47$ to 0.78$)$ (Figure $7 \mathrm{~A})$. As for CD, meta-analysis also showed significant difference of RR $(0.33,95 \% \mathrm{Cl} 0.15$ to 0.76$)$ (Figure 7B). 5 of the RCTs had also documented the LVEF after intervention. Data was pooled together and synthetized, the results favored TCM group (SMD 0.94, 95\% $\mathrm{Cl} 0.22$ to 1.66) (Figure 7C). Research had shown that the 30-day cardiac mortality rate in ST elevated myocardial infarction (STEMI) patients was $7.3 \%$ even underwent $\mathrm{PCl}$, the 1 -year cardiac mortality rate was $8.4 \%$, with a $<1.5 \%$ annual risk of successive cardiac death ${ }^{15}$. To reduce the short-term cardiac mortality rate in AMI patients is vital to control the over-all outcomes of MI patients. Cardiac mortality rate in TCM group is $4.1 \%$. The results in these research indicated that TCM may be a key role in this goal.

\section{TCM for PMI}

Post myocardial infarction is usually reckoned as 4 to 8 weeks after acute myocardial infarction. If the patient survive from AMI and/or cardiac shock, malignant arrhythmia, cardiac remodeling and coronary collateral circulation establishment should be the major physiopathological process ${ }^{16,17}$. Prompt and adequate intervention can boost the cardiac rehabilitation and prolong life expectancy ${ }^{18} .7$ RCTs (No.5561) evaluated TCM treating PMI were included and assessed. All the participants experienced AMI 1-12 months before inclusion, despite recanalization or not. The sample size ranged from 60 to 4870 participants, with average of 763.57 (average of 79.17 if the largest scale RCT was ruled out). The intervention duration ranged from 4 to 216 weeks, with average of 42.29 weeks (average of 13.33 if the largest scale RCT was ruled out). The methodological quality of the included RCTs was generally low. 1 of 7 RCTs had a Jadad score of 5,1 had a Jadad score of 3, and 5 had score of 2 . The major and secondary outcomes we included and assessed were clinical efficacy, angina efficacy, LVEF and MACE.

5 of 7 RCTs had reported the clinical efficacy after intervention. All of them compared TCM with blank intervention, with the basis of antiplatelet, beta blocker, statins, ACEI/ARB and nitrates. Meta-analysis had shown significant difference of $\mathrm{RR}(1.28,95 \% \mathrm{Cl}, 1.15$ to 1.42$)$. Statistical heterogeneity in this model was considered low ( $P=0.76, \mathrm{I}^{2}=0 \%$ ) (Figure $8 \mathrm{~A}$ ). $3 \mathrm{RCT}$ sere included in the meta-analysis of angina efficacy, and had shown significant difference of $\mathrm{RR}(1.48,95 \% \mathrm{Cl}, 1.23$ to 1.78$)$. Statistical heterogeneity in this model was considered low $\left(P=0.26, \mathrm{I}^{2}=25 \%\right)$ (Figure $\left.8 \mathrm{~B}\right)$. This result indicated that TCM can improve the clinical and angina efficacy of PMI.

LVEF reflects the pumping function of left ventricular. Size of infarct myocardium, formation of ventricular aneurysm, ventricular remodeling, arrhythmia and cardiac rehabilitation are the detrimental and beneficial factors of remaining LVEF. 3 RCTs documented LVEF after intervention. Meta-analysis showed that in LVEF, TCM did not achieve significant progression compared to blank intervention (RR $0.36,95 \% \mathrm{Cl}-0.08$ to 0.80 ) (Figure $9 \mathrm{~A}$ ). As for MACE in PMI after intervention, the incidence rate in TCM group was $5.18 \%$, compared to $8.04 \%$ in control group (RR $0.56,95 \% \mathrm{Cl} 0.32$ to 0.97 ) (Figure 9B). TCM has significant effect on reducing incidence rate of MACE. 


\section{TCM for CHF}

As more patients survive and live longer after $\mathrm{MI}$, the incidence and prevalence of IHD related CHF continue to rise ${ }^{19}$. Cardiovascular and non-cardiovascular caused HF will have different pathologies. In patients with HF (both hospitalized and ambulatory), most deaths are due to cardiovascular causes ${ }^{20}$. 16 RCTs (No. 62-77) evaluated TCM treating CHF were included and assessed. All the participants in every RCTs were diagnosed IHD or previous MI with formally published criteria. The sample size ranged from 60 to 491 participants, with average of 110.69. The intervention duration ranged from 4 to 24 weeks, with average of 9.25 weeks. The methodological quality of the included RCTs was considered moderate to low. Only 1 of 16 RCTs had a Jadad score of 5 and 3, the rest 14 RCTs had score of 2 . The major and secondary outcomes we included and assessed were clinical efficacy, $\mathrm{N}$ terminal pro B type natriuretic peptide (NT-proBNP), LVEF and 6-minute walk test (6MWT), according to the data in RCTs we included.

13 of 16 RCTs had reported the clinical efficacy after intervention. All of them compared TCM with blank intervention, with the common basis of diuretic, Aldosterone antagonists, beta blocker and ACEI/ARB. Meta-analysis had shown significant difference of $\mathrm{RR}(1.23,95 \% \mathrm{Cl}, 1.13$ to 1.34$)$. Statistical heterogeneity in this model was considered high $\left(P=0.002, I^{2}=61 \%\right)$ (Figure 10). This result indicated that TCM can improve the clinical efficacy of CHF.

6 RCTs had documented the level of NT-proBNP in each group after intervention. Meta-analysis showed significant reduction of SMD in TCM group (SMD -2.72, 95\% Cl -4.26 to -1.19) (Figure 11A). As for LVEF and 6MWT, 16 RCTs and 10 RCTs documented LVEF and 6MWT respectively, meta-analysis showed significant increase of SMD in TCM groups (LVEF: SMD 0.91, 95\% Cl 0.45 to 1.37; 6MWT: SMD 0.65, 95\% $\mathrm{Cl} 0.41$ to 0.89 ) (Figure 11B and 11C). NT-proBNP and LVEF can serve as ideal parameters in diagnosing and evaluating severity of $\mathrm{HF}$, also associated with increased mortality and morbidity in patients with $\mathrm{HF}^{21,22}$. And the 6MWT independently predicts CHF severity, hospitalization and death ${ }^{23}$. TCM may ameliorate clinical symptoms and improve life quality in patients with CHF.

\section{Discussion}

Clinical efficacy, or the amelioration of symptoms, is the major purpose and evaluation of TCM intervention, according to its nautural characteristics. It is the ability to relieve clinical symptoms that makes it survive and prosper for more than 2 thousand years. Modern TCM has embraced advanced modern medicine and science for more than 2 hundred years. One of the pharmaceutical monograph named < Bencaogangmu Shiyi> (supplementto compendium of materia medica) has recorded quinine as imported product for the treatment of malaria back in 1765. In 1909, one of the famous clinical monograph named < Yixue Zhongzhong Canxilu> (practical records of traditional Chinese medicine with reference to western medicine) had presented a formula with aspirin as one of the ingredients for the treatment of flu. Till mid-20th century, the clinical practice and research of real modern TCM was initially formed ${ }^{24}$. Interestingly, the frontier aspect of modern TCM research was CHD. Decades have passed by and we have learned that TCM can both ameliorate clinical symptoms and improve objective parameters. 
More and more clinical and experimental evidence has been revealed to support the utilization of TCM as primary or complementary therapy for diseases.

The quantification of TCM symptoms was once obstacle for the evaluation and promotion of TCM. The Guiding Principles for Clinical Research of New Chinese Medicines (published by State Drug Administration of China in 2002) has uniformed the chaos of clinical efficacy evaluation. It was based on patient self-reported severity of various clinical symptoms to form a disease assessment scale. The symptoms used in evaluation varies in different diseases, and are most related to the concern of the diseases. With this equipment allows researchers assess the clinical efficacy in a more quantitative way, especially in some diseases which clinical manifestations play important role in the management and patient satisfaction (i.e. SA, CHF with NYHA class $₫ / \otimes$ cardiac function) ${ }^{25,26}$.

As we mentioned above, we value the clinical efficacy comparison after intervention. 'Effective' was defined as the total score of symptoms reduced over $50 \%$ after intervention in the RCTs we included. Of all the aspects of IHD this research concerned, TCM achieved better clinical efficacy, with RR ranged between 1.14 to 1.30 . The evaluation and the results of angina efficacy were similar to clinical efficacy in these analyses, and were corroborated by the analyses of angina frequency and nitrates consumption. Considering almost every RCT was conducted on the basis of conventional treatment in both TCM group and control group, it is safe to say that TCM can improve clinical efficacy as complementary to western medicine. The improvement of clinical efficacy was coincided with previous reports ${ }^{25-30}$.

As the clinical efficacy was improved, objective parameters were altered in these RCTs. In different stages of IHD, the secondary outcomes, or what we pay more attention to, is different. Such as in SA, patients experience occasional angina pectoris, apart from angina relieving and controlling, the management of $S A$ is control of risk factors. Since in this research we mainly focus on the IHD, the risk factor in this aspect was dyslipidemia. Meta-analyses had shown that TCM could lower levels of TC, LDL-C, TG and increase level of HDL-C in SA. These can gain dependable benefit for patients with SA in the holistic management of IHD.

The secondary outcomes we synthesized and analyzed were affected by RCTs we included. In the aspect of UA, only LDL-C was pooled and analyzed, as seldom study documented other types of lipid. And CRP was meta-analyzed, since UA is the unstable form of atherosclerosis with increased level of inflammation ${ }^{31}$, CRP was tested as useful biomarker. In the aspect of AMI and PMI, MACE and CD were more important to assess the value of TCM intervention. MACE and CD are more likely to happen closer to the onset of $\mathrm{MI}^{15}$. It is vital to control recurrent cardiovascular events in patients experienced MI. These analyses indicated that TCM can reduce the incidence rate of MACE and CD both in acute and non-acute stage of Ml.

LVEF reflects the pumping function of left ventricular. Local myocardial damage or long-term myocardium ischemia can lead to the reduction of LVEF. LVEF was documented in AMI, PMI and CHF related RCTs. Level of LVEF is one of the major diagnostic and stratification biomarkers for HF. All-cause 
mortality is generally higher in $\mathrm{HF}$ with reduced $\mathrm{EF}(\mathrm{HFrEF})$ than $\mathrm{HF}$ with preserved $\mathrm{EF}(\mathrm{HFpEF})^{32}$. Compared with patients with HFpEF and patients with HFrEF, patients with HF with recovered EF (HFrecEF) had fewer all-cause, cardiovascular and HF-related hospitalizations and were less likely to experience composite end points ${ }^{33}$. NT-proBNP have diagnostic and prognostic value in patients with $\mathrm{HF}^{34}$. Reduction of NT-proBNP is associated with reduced morbidity and mortality outcomes, whereas increased NT-proBNP portends poor patient outcomes ${ }^{35}$. The 6MWT has important prognostic value in patients with chronic heart failure ${ }^{36}$. It has also been recommended to monitor the disease course and to assess the efficacy of intervention in patients with mild-to-moderate $\mathrm{CHF}^{37}$. Improvement of 6MWT contributed to improvement of exercise confidence in $\mathrm{HF}$ patients, which indicated more intention to perform exercise ${ }^{38}$. Meta-analyses of LVEF in AMI, and CHF, and of NT-proBNP and 6MWT in CHF had provided evidence for the TCM potential long-term protection for IHD.

Systematic review and meta-analysis of TCM are popular for years, because the screening and synthesizing process can enhance the reliability of TCM RCTs, which mostly were moderate to low quality. Previously published meta-analyses had provided beneficial evidence for single TCM medication in some aspects of IHD respectively. Some of them had also taken IHD or CHD as whole to assess the efficacy of TCM. Panpan Hao et al. ${ }^{39}$ had evaluated TCM therapy for several CVD risk factors, atherosclerotic cardiovascular disease (ASCVD) and CHF in 1 study. They included RCTs published in the past decade with a relatively strict criteria. Subgroup analyses showed the landscape of TCM therapy in different aspects of CVD. The methodological quality of included RCTs were moderate to high, the results and conclusions of this review are considered reliable. Meanwhile they only included RCTs with objective laboratory measurements or physical examination, clinical symptoms improvement evaluation was absent from this study. In this high quality, wide spectrum systematic review of TCM therapy for CVD, it would have been intriguing to evaluate if TCM could improve clinical efficacy along with the amelioration of objective parameters. Another systematic review had also evaluated TCM in the management of some of the most important cardiovascular diseases, including hypertension, acute ischemic stroke, HF, CHD and type 2 diabetes ${ }^{40}$. In this study, hard endpoint was required, too. But the methodology quality was not restricted in the inclusion criteria. The results were similar to Panpan Hao et al. in certain aspects.

The purpose of our study is to evaluate the efficacy of TCM in different stages of IHD. TCM is now widely used in the treatment of CVD in the eastern Asia, with the tendency of worldwide. It is urged to demonstrate more convincing evidence for the clinical practice of TCM. We divided IHD into 5 stages: SA, UA, AMI, PMI, HF, in which TCM are usually applied and more applicable for systematic analysis. These stages represent the developing process of IHD. In stage of SA, CVD risk factors are more likely to be managed, the control of angina symptom can be a challenge in clinical practice. In stage of UA and AMI, guidelines for acute coronary syndrome are elaborate, recanalization is sometimes needed. Any approach to reduce the mortality rate is encouraged. When patient has survived $\mathrm{Ml}$, reducing recurrence of cardiovascular events and preserving cardiac function would be the priority in clinical practice. By the meta-analyses in this study, we have failed in locating the best breakthrough point of TCM for IHD, but 
have discovered that TCM should be used in all these stages of IHD as complementary medicine to better the clinical outcomes.

Studies both in English and in Chinese were screened and included. The methodology quality of RCTs we included was considered moderate, as we ruled out all the RCTs with Jadad score less than 2, those without definite randomization method were excluded, either. Primary and secondary endpoints we included and analyzed were based on the disease features and the included RCTs.

There were some potential limitations and sources of variability to this review: 1) The individual RCTs differed in baseline characteristics, especially some baseline parameters such as NT-proBNP, LVEF, and the baseline western medication. 2) The follow-up duration was moderate to short, the sample size was relatively small. 3) Heterogeneity was considered moderate to high between studies in some of the comparison. Baseline, follow-duration and sample size inconsistence may contribute to it. 4) Most of the RCTs compared TCM with blank intervention, only a few had used placebo. TCM decoction and some patent medicine are mostly highly-scented, it is difficult to produce satisfying placebo. 5) Non-drug therapy was not included in this study, such as acupuncture and moxibustion, Taichi, massage. The combination of drug therapy and non-drug therapy would increase the Heterogeneity. To eliminate these limitations and enhance the reliability, further rigorously designed RCTs with larger scale and multiple centers are needed.

\section{Conclusions}

Our meta-analysis of data from RCTs supports the use of TCM in different stages of IHD. TCM can both improve the clinical manifestations and ameliorate the objective parameters. Some of the improvements lead to potential long-term benefits. To acquire more solid and comprehensive evidence of TCM in treating CVD, larger scaled, multi-centered, double-blinded RCTs with reliable placebo and longer follow-up duration are warranted.

\section{Abbreviations}




\begin{tabular}{ll} 
6MWT & 6-minute walk test \\
\hline ACS & acute coronary syndromes \\
\hline AMI & acute myocardial infarction \\
\hline ASCVD & atherosclerotic cardiovascular disease \\
\hline CABG & coronary artery bypass grafting \\
\hline CD & cardiovascular death \\
\hline CHF & chronic heart failure \\
\hline Cl & confidence interval \\
\hline CRP & C-reactive protein \\
\hline CVD & Cardiovascular diseases \\
\hline GBD & Global Burden of Death \\
\hline HDL-C & high density lipoprotein-cholesterol \\
\hline HFpEF & heart failure with preserved ejection fraction \\
\hline HFrecEF & heart failure with recovered ejection fraction \\
\hline HFrEF & heart failure with reduced ejection fraction \\
\hline IHD & ischemic heart disease \\
\hline LDL-C & low density lipoprotein-cholesterol \\
\hline LVEF & left ventricular ejection fraction \\
\hline MACE & major adverse cardiovascular events \\
\hline NT-proBNP & N terminal pro B type natriuretic peptide \\
\hline PCI & percutaneous coronary intervention \\
\hline PMI & post myocardial infarction \\
\hline RR & relative risk \\
\hline SA & stable angina \\
\hline SIHD & stable ischemic heart disease \\
\hline SMD & standard mean difference \\
\hline STEMI & ST elevated myocardial infarction \\
\hline TC & total cholesterol \\
\hline Traditional Chinese medicine \\
\hline Her
\end{tabular}




$\begin{array}{ll}\text { TG } & \text { triglyceride } \\ \text { UA } & \text { unstable angina }\end{array}$

\section{Declarations}

\section{Acknowledgements}

Not applicable.

\section{Author contributions}

$\mathrm{JL}, \mathrm{XL}$ and JD designed and supervised the study. JL and JT carried out the search and screening criteria. MS, ZW, KW and JC performed literature search, screening and data collection. JL, JT drafted the manuscript and $X L$, JD revised it.

\section{Funding}

This work was supported by the National Science Foundation of China (No. 81803923, No. 81873138), Young Elite Scientists Sponsorship Program by CAST (2018QNRC2-C10, 2017QNRC1-02), Science Research Fund of China-Japan Friendship Hospital (No. 2017-2-QN-13).

\section{Availability of data and materials}

The datasets used and/or analysed during the current study are available from the corresponding author on reasonable request.

\section{Ethics approval and consent to participate}

Not applicable.

\section{Consent for publication}

Not applicable.

\section{Competing interests}

The authors declare that they have no competing interests.

\section{References}

1. GBD 2013 Mortality and Causes of Death Collaborators. Global, regional, and national age-sex specific all-cause and cause-specific mortality for 240 causes of death, 1990-2013: a systematic analysis for the Global Burden of Disease Study 2013. Lancet. 2015;385(9963):117-71. 
2. GBD 2015 Mortality and Causes of Death Collaborators. Global, regional, and national life expectancy, all-cause mortality, and cause-specific mortality for 249 causes of death, 1980-2015: a systematic analysis for the Global Burden of Disease Study 2015. Lancet. 2016; 388(10053): 14591544.

3. Roth GA, Johnson C, Abajobir A, et al. Global, regional, and national burden of cardiovascular diseases for 10 causes, 1990 to 2015. J Am Coll Cardiol. 2017;70(1):1-25.

4. University of Washington Institute of Health Metrics and Evaluation. GBD Compare VizHub. 2017. Available at: https://vizhub.healthdata.org/gbd-compare/. Accessed December 17, 2018.

5. Martel J, Ojcius DM, Chang CJ, et al. Anti-obesogenic and antidiabetic effects of plants and mushrooms. Nat Rev Endocrinol. 2017;13(3):149-160.

6. Chang CJ, Lin CS, Lu CC, et al. Ganoderma lucidum reduces obesity in mice by modulating the composition of the gut microbiota. Nat Commun. 2015;6:7489.

7. Li X, Zhang J, Huang J, et al. A multicenter, randomized, double-blind, parallel-group, placebocontrolled study of the effects of qili qiangxin capsules in patients with chronic heart failure. J Am Coll Cardiol. 2013;62(12):1065-1072.

8. Normile D. Asian medicine. The new face of traditional Chinese medicine. Science. 2003;299(5604):188-90.

9. Efferth T, Kaina B. Toxicities by herbal medicines with emphasis to traditional Chinese medicine. Curr Drug Metab 2011; 12: 989-96.

10. Wu X, Tang J, Mao C, et al. Systematic reviews and meta-analyses of traditional chinese medicine must search chinese databases to reduce language bias. Evid Based Complement Alternat Med. 2013;2013:812179.

11. Jadad AR, Moore RA, Carroll $D$, et al. Assessing the quality of reports of randomized clinical trials: is blinding necessary? Control Clin Trials. 1996;17(1):1-12.

12. Review Manager (RevMan) Computer program.. Version 5.3. Copenhagen: The Nordic Cochrane Centre, The Cochrane Collaboration, 2014.

13. Patel MR, Calhoon JH, Dehmer GJ, et al. ACC/AATS/AHA/ASE/ASNC/SCAI/SCCT/STS 2017 Appropriate Use Criteria for Coronary Revascularization in Patients With Stable Ischemic Heart Disease: A Report of the American College of Cardiology Appropriate Use Criteria Task Force, American Association for Thoracic Surgery, American Heart Association, American Society of Echocardiography, American Society of Nuclear Cardiology, Society for Cardiovascular Angiography and Interventions, Society of Cardiovascular Computed Tomography, and Society of Thoracic Surgeons. J Am Coll Cardiol. 2017;69(17):2212-2241.

14. Piepoli MF, Hoes AW, Agewall S, et al. ESC Scientific Document Group. 2016 European Guidelines on cardiovascular disease prevention in clinical practice: The Sixth Joint Task Force of the European Society of Cardiology and Other Societies on Cardiovascular Disease Prevention in Clinical Practice (constituted by representatives of 10 societies and by invited experts)Developed with the special 
contribution of the European Association for Cardiovascular Prevention \& Rehabilitation (EACPR). Eur Heart J. 2016;37(29):2315-2381.

15. Pedersen $F$, Butrymovich $\mathrm{V}$, Kelbæk $\mathrm{H}$, et al. Short- and long-term cause of death in patients treated with primary PCI for STEMI. J Am Coll Cardiol. 2014;64(20):2101-8.

16. Pfeffer MA, Braunwald E. Ventricular remodeling after myocardial infarction. Experimental observations and clinical implications. Circulation. 1990;81(4):1161-72.

17. Hirt MN, Hansen A, Eschenhagen T. Cardiac tissue engineering: state of the art. Circ Res. 2014;114(2):354-67.

18. Bahit MC, Kochar A, Granger CB. Post-Myocardial Infarction Heart Failure. JACC Heart Fail. 2018;6(3):179-186.

19. van Riet EE, Hoes AW, Wagenaar KP, et al. Epidemiology of heart failure: the prevalence of heart failure and ventricular dysfunction in older adults over time. A systematic review. Eur J Heart Fail. 2016;18(3):242-52.

20. Ponikowski P, Voors AA, Anker SD, et al. ESC Scientific Document Group. 2016 ESC Guidelines for the diagnosis and treatment of acute and chronic heart failure: The Task Force for the diagnosis and treatment of acute and chronic heart failure of the European Society of Cardiology (ESC)Developed with the special contribution of the Heart Failure Association (HFA) of the ESC. Eur Heart J. 2016;37(27):2129-2200.

21. Hartmann F, Packer M, Coats AJ, et al. Prognostic impact of plasma N-terminal pro-brain natriuretic peptide in severe chronic congestive heart failure - A substudy of the carvedilol prospective randomized cumulative survival (COPERNICUS) trial. Circulation. 2004;110(13):1780-6.

22. Vasan RS, Xanthakis V, Lyass A, et al. Epidemiology of Left Ventricular Systolic Dysfunction and Heart Failure in the Framingham Study An Echocardiographic Study Over 3 Decades. JACC Cardiovasc Imaging. 2018;11(1):1-11.

23. Forman DE, Fleg JL, Kitzman DW, et al. 6-min walk test provides prognostic utility comparable to cardiopulmonary exercise testing in ambulatory outpatients with systolic heart failure. J Am Coll Cardiol. 2012;60:2653-61.

24. Liao J, Wang J, Liu Y, et al. Modern researches on Blood Stasis syndrome 1989-2015: A bibliometric analysis. Medicine (Baltimore). 2016;95(49):e5533.

25. Jia $Y$, Huang F, Zhang $S$, et al. Is danshen (Salvia miltiorrhiza) dripping pill more effective than isosorbide dinitrate in treating angina pectoris? A systematic review of randomized controlled trials. Int J Cardiol. 2012;157(3):330-40.

26. Zhang Y, Xie Y, Liao X, et al. A Chinese patent medicine Salvia miltiorrhiza depside salts for infusion combined with conventional treatment for patients with angina pectoris: A systematic review and meta-analysis of randomized controlled trials. Phytomedicine. 2017;25:100-117.

27. Wang KH, Wu JR, Zhang D, et al. Comparative efficacy of Chinese herbal injections for treating chronic heart failure: a network meta-analysis. BMC Complement Altern Med. 2018;18(1):41. 
28. Chen $\mathrm{R}$, Xiao $\mathrm{Y}$, Chen $\mathrm{M}$, et al. A traditional Chinese medicine therapy for coronary heart disease after percutaneous coronary intervention: a meta-analysis of randomized, double-blind, placebo-controlled trials. Biosci Rep. 2018;38(5). pii: BSR20180973.

29. Liao P, Wang L, Guo L, et al. Danhong Injection (a Traditional Chinese Patent Medicine) for Acute Myocardial Infarction: A Systematic Review and Meta-Analysis. Evid Based Complement Alternat Med. 2015;2015:646530.

30. Fu S, Zhang J, Menniti-Ippolito F, et al. Huangqi injection (a traditional Chinese patent medicine) for chronic heart failure: a systematic review. PLoS One. 2011;6(5):e19604.

31. Liuzzo G, Biasucci LM, Gallimore JR, et al. The prognostic value of C-reactive protein and serum amyloid a protein in severe unstable angina. N Engl J Med. 1994;331(7):417-24.

32. Maggioni AP, Dahlstro“m U, Filippatos G, et al. EURObservational Research Programme: regional differences and 1-year follow-up results of the Heart Failure Pilot Survey (ESC-HF Pilot). Eur J Heart Fail 2013;15:808-817.

33. Kalogeropoulos AP, Fonarow GC, Georgiopoulou V, et al. Characteristics and Outcomes of Adult Outpatients With Heart Failure and Improved or Recovered Ejection Fraction. JAMA Cardiol. 2016;1(5):510-8.

34. Savarese G, Musella F, D'Amore C, et al. Changes of natriuretic peptides predict hospital admissions in patients with chronic heart failure: a meta-analysis. JACC Heart Fail. 2014;2(2):148-58.

35. Zile MR, Claggett BL, Prescott MF, et al. Prognostic Implications of Changes in N-Terminal Pro-B-Type Natriuretic Peptide in Patients With Heart Failure. J Am Coll Cardiol. 2016;68(22):2425-2436.

36. Bittner $\mathrm{V}$, Weiner $\mathrm{DH}$, Yusuf $\mathrm{S}$, et al. Prediction of mortality and morbidity with a 6-minute walk test in patients with left ventricular dysfunction. SOLVD investigators. JAMA. 1993; 270: 1702-1707.

37. Uszko-Lencer NHMK, Mesquita R, Janssen E, et al. Reliability, construct validity and determinants of 6-minute walk test performance in patients with chronic heart failure. Int J Cardiol. 2017;240:285290.

38. Ha F, Toukhsati SR, Cameron JD, et al. Association between the 6-minute walk test and exercise confidence in patients with heart failure: A prospective observational study. Heart Lung. 2018;47(1):54-60.

39. Hao P, Jiang F, Cheng J, et al. Traditional Chinese Medicine for Cardiovascular Disease: Evidence and Potential Mechanisms. J Am Coll Cardiol. 2017;69(24):2952-2966.

40. Layne K, Ferro A. Traditional Chinese medicines in the management of cardiovascular diseases: a comprehensive systematic review. Br J Clin Pharmacol. 2017;83(1):20-32.

\section{Tables}

Due to technical limitations, Table 1 is only available as a download in the supplemental files section

\section{Figures}




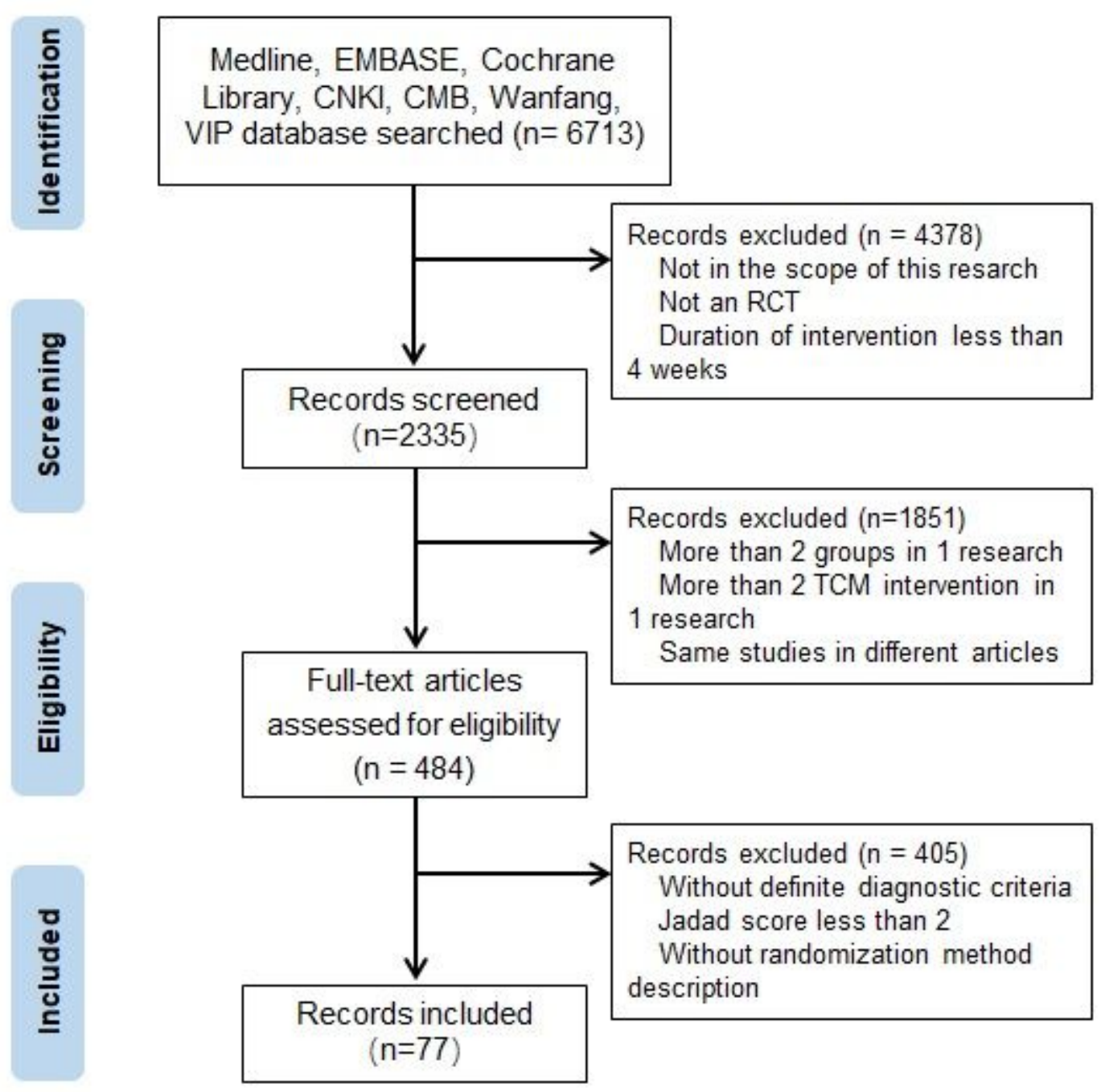

\section{Figure 1}

Flow diagram of the RCT inclusion process. 
A

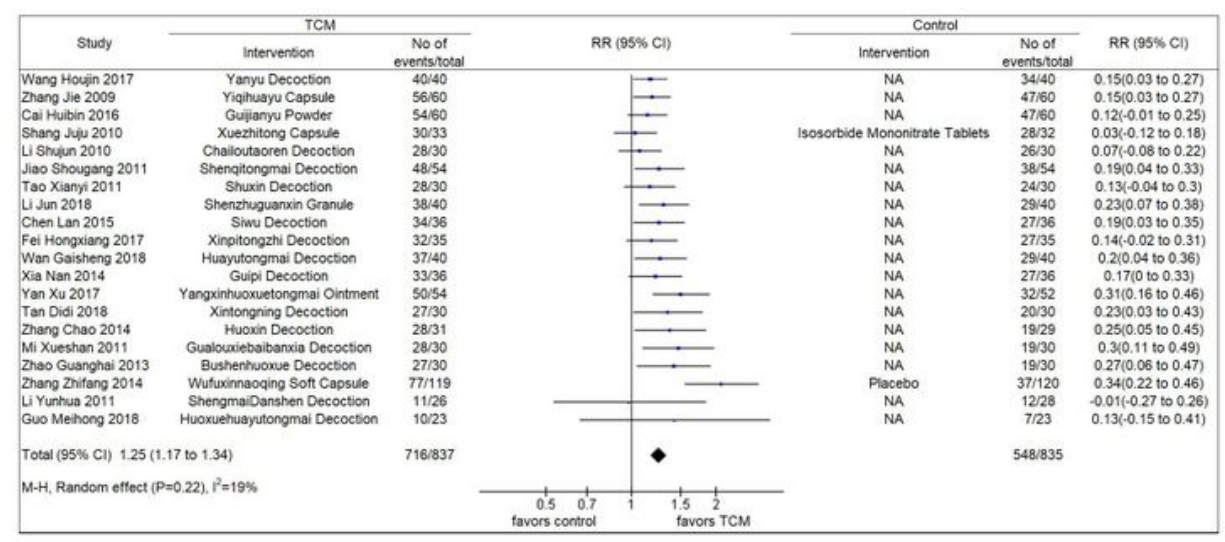

B

\begin{tabular}{|c|c|c|c|c|c|c|c|}
\hline \multirow{2}{*}{ study } & \multicolumn{2}{|l|}{ TCM } & \multirow{2}{*}{\multicolumn{2}{|c|}{$R R(95 \% \mathrm{Cl})$}} & \multicolumn{2}{|l|}{ control } & \multirow[b]{2}{*}{$R R(95 \% \mathrm{Cl})$} \\
\hline & Intervention & $\begin{array}{l}\text { No of } \\
\text { eventshotal }\end{array}$ & & & Imtervention & $\begin{array}{l}\text { Noo of } \\
\text { ventshotal }\end{array}$ & \\
\hline 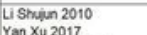 & 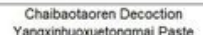 & $\begin{array}{l}28330 \\
4945\end{array}$ & & & & 26630 & $1.08(0.91$ to 1.28$)$ \\
\hline $\begin{array}{l}\text { Yan Xu } 20171 \\
\text { Shang Jiju } 2010\end{array}$ & 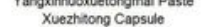 & $\begin{array}{l}4954 \\
29333\end{array}$ & & E & Isosorbibse Monorntate Tablets & $\begin{array}{l}39522 \\
2832\end{array}$ & $\begin{array}{l}1.21(1.01101 .45) \\
1084101.21)\end{array}$ \\
\hline Zhang Jie 2009 & Yiqhuayu Capsule & 54150 & & $\longrightarrow$ & NA & 4260 & $1.29(1.07$ to 1.55$)$ \\
\hline $\begin{array}{l}\text { Wan Gashseng } 2018 \\
\text { Tan Dadi ing }\end{array}$ & $\begin{array}{l}\text { Huarytongmai Decoction } \\
\text { Xintongning gecoction }\end{array}$ & $\begin{array}{l}37400 \\
27730\end{array}$ & & $\because$ & ${ }_{N A}^{N A}$ & $\begin{array}{l}30 / 40 \\
23 / 30\end{array}$ & $\begin{array}{l}1.231 .01101 .51 \\
1.1709310148\end{array}$ \\
\hline Gao Jianwei 2018 & Xinling pil & $90 / 114$ & & & $\begin{array}{l}\text { Pacebo } \\
\text { Pace }\end{array}$ & $49 / 115$ & 1.85(1.47 102.34) \\
\hline $\begin{array}{l}\text { Mixuesthan } 2011 \\
\text { Xie e Jing } 2015\end{array}$ & $\begin{array}{l}\text { Guallowiebabibancia decoction } \\
\text { Xingling tablets }\end{array}$ & 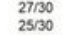 & & $\div$ & ${ }_{N A}^{N A}$ & ${ }_{23350}^{2230}$ & $\begin{array}{l}1.230 .96601 .157) \\
1.09(1.8440 .14)\end{array}$ \\
\hline $\begin{array}{l}\text { Fei Hongoviang } 2017 \\
\text { Zhemp Chao } 2014\end{array}$ & $\begin{array}{l}\text { Xnpitongerti Decoction } \\
\text { Hwo }\end{array}$ & $\begin{array}{l}3035 \\
2701 \\
2701\end{array}$ & & . & NA & 2335 & $1.3(0.99$ to 1.72$)$ \\
\hline & & & & & & & \\
\hline \multicolumn{2}{|c|}{ Total $(95 \%$ Cl) $1.23(1.11$ to 1.37 ) } & 42344877 & & - & & 3234483 & \\
\hline \multicolumn{3}{|c|}{$M-H$, Random etfect $(P=0.005), P^{2}=60 \%$} & $\begin{array}{l}0.5 \\
0.5 \\
\text { tavors control }\end{array}$ & 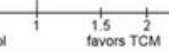 & & & \\
\hline
\end{tabular}

C

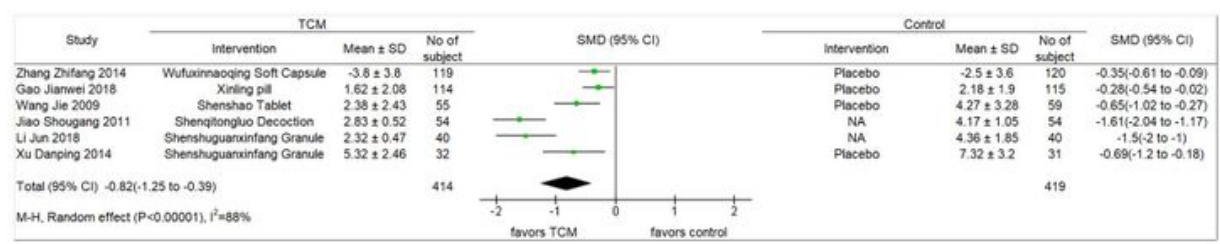

D

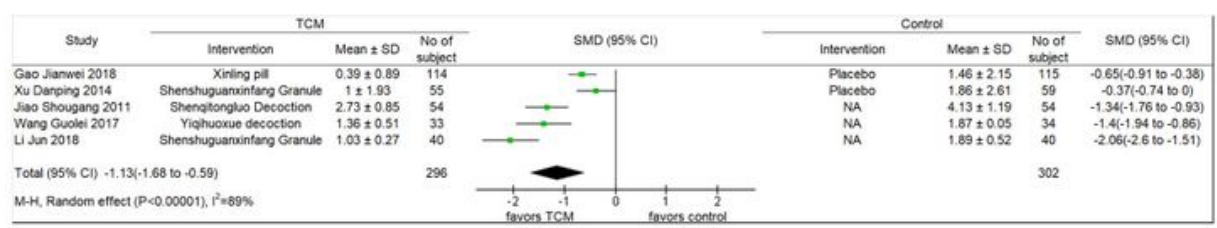

E

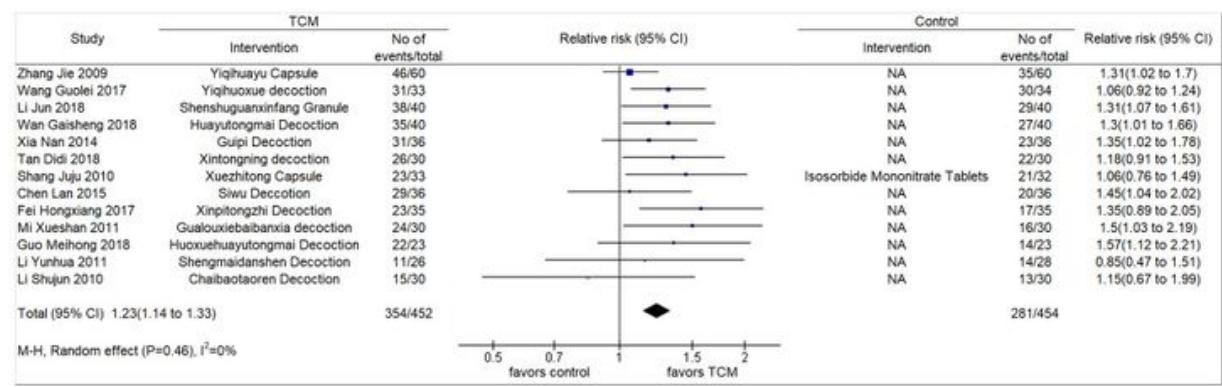

Figure 2

Clinical and angina efficacy comparison between TCM group and control group in SA. A: Clinical efficacy comparison between TCM group and control group. B: Angina efficacy comparison between TCM group and control group. C: Angina frequency comparison between TCM group and control group. D: Nitrates consumption comparison between TCM group and control group. E: ECG comparison between TCM group and control group. 
A

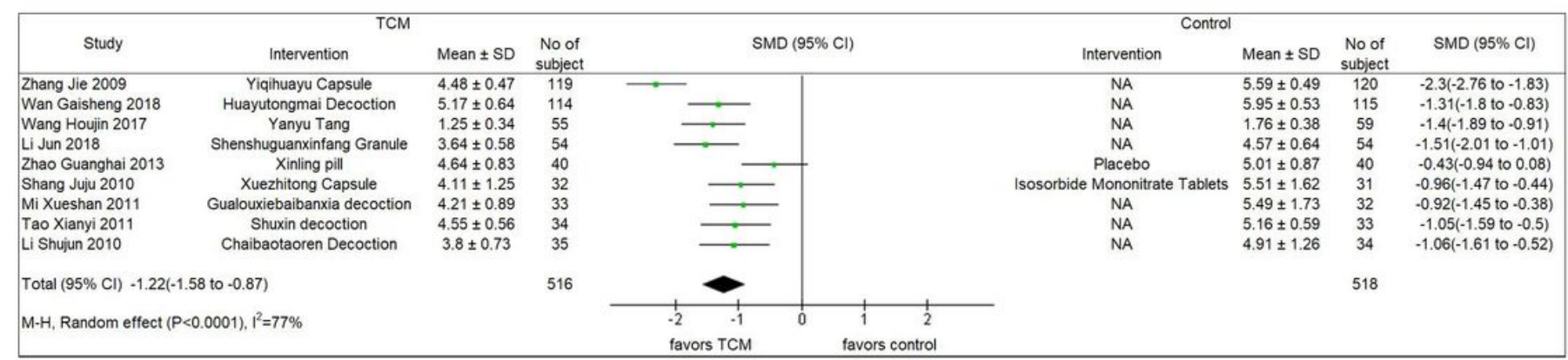

B

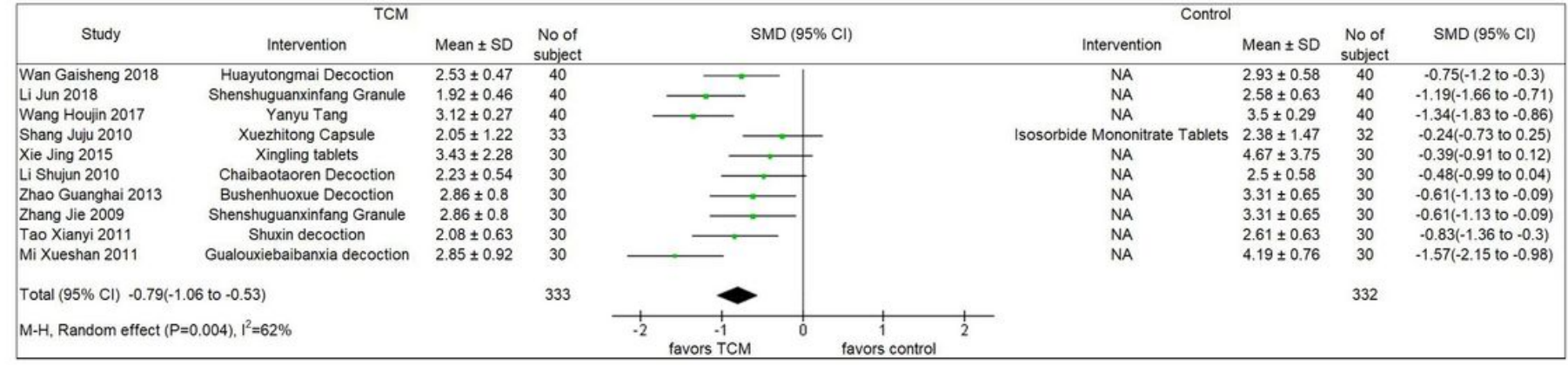

C

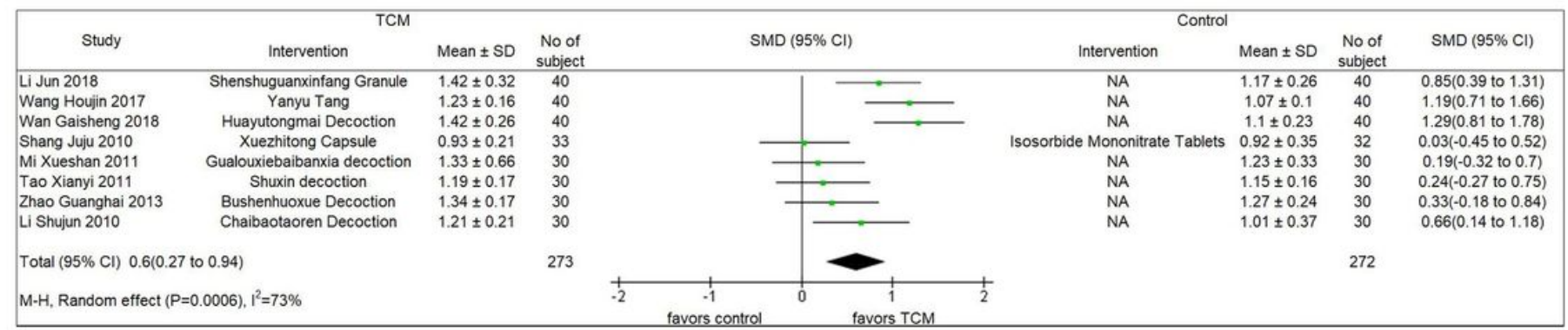

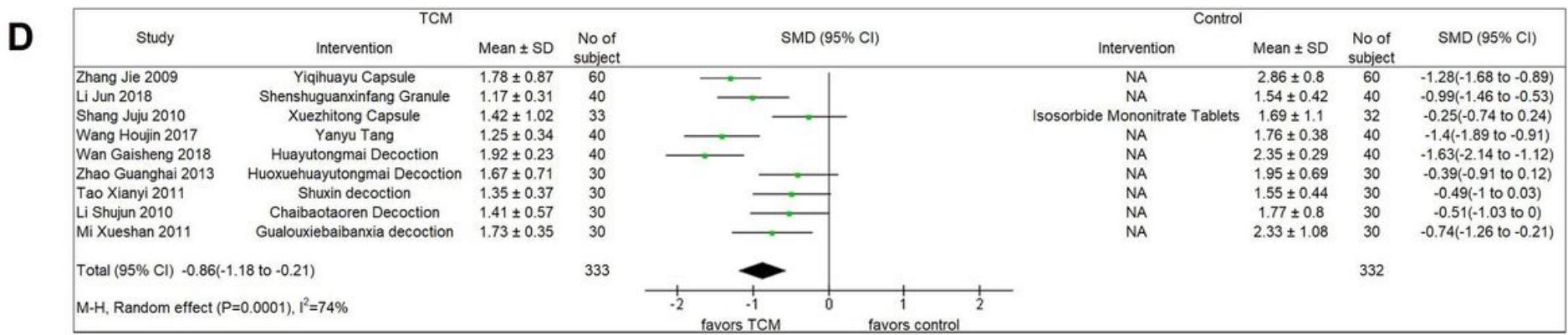

\section{Figure 3}

Lipids comparison between TCM group and control group in SA. A: TC comparison between TCM group and control group. B: LDL-C comparison between TCM group and control group. C: HDL-C comparison between TCM group and control group. D: TG comparison between TCM group and control group. 
A

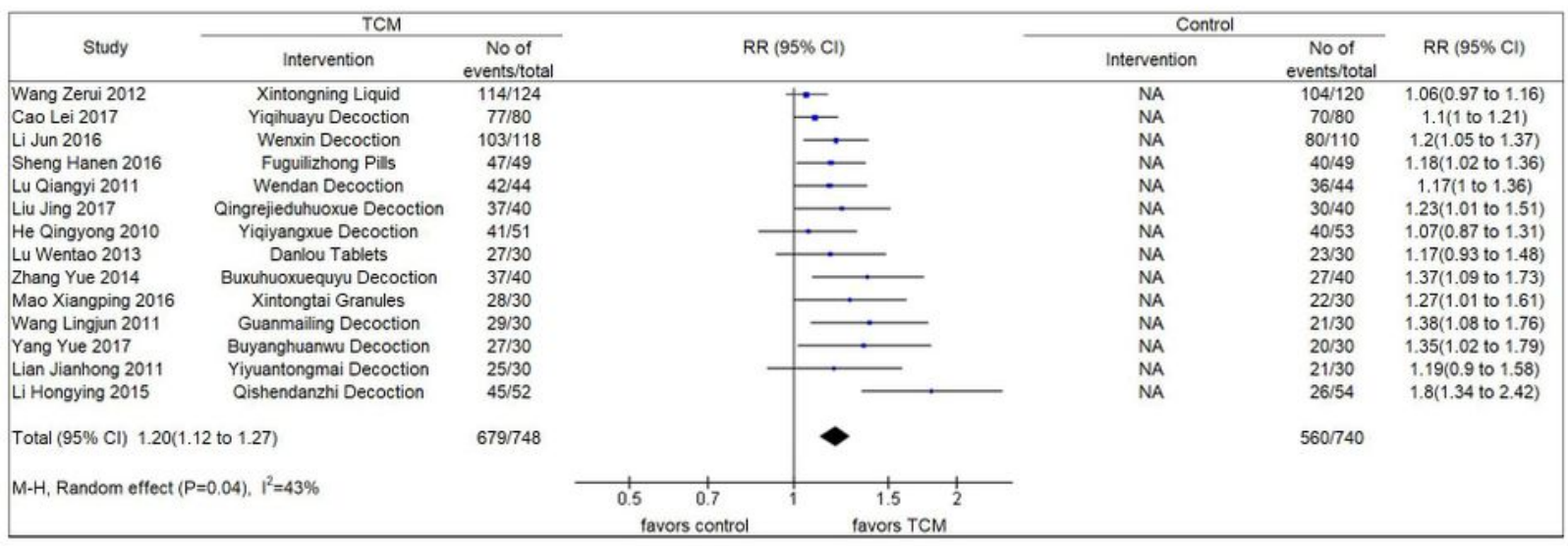

B

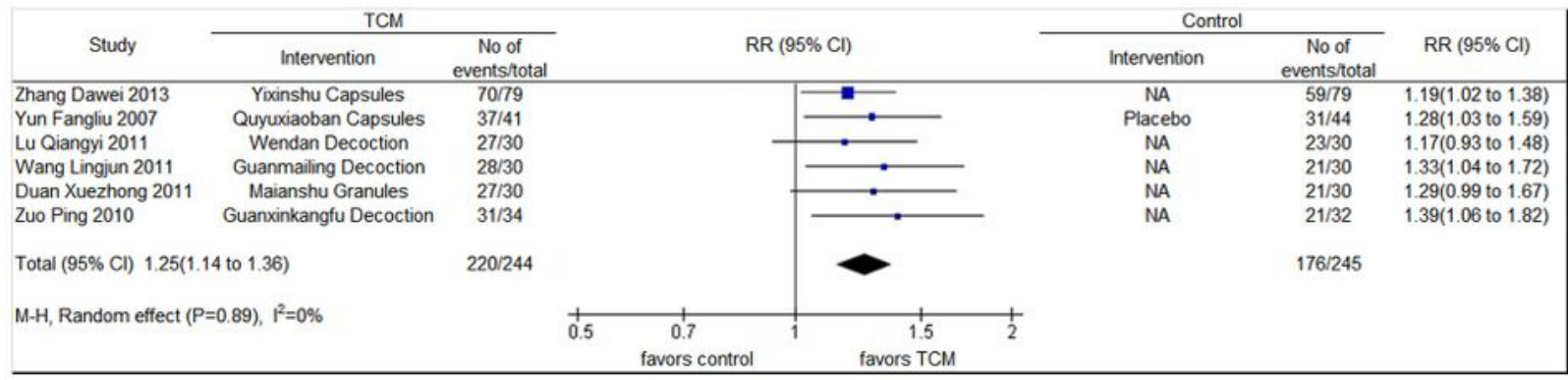

C

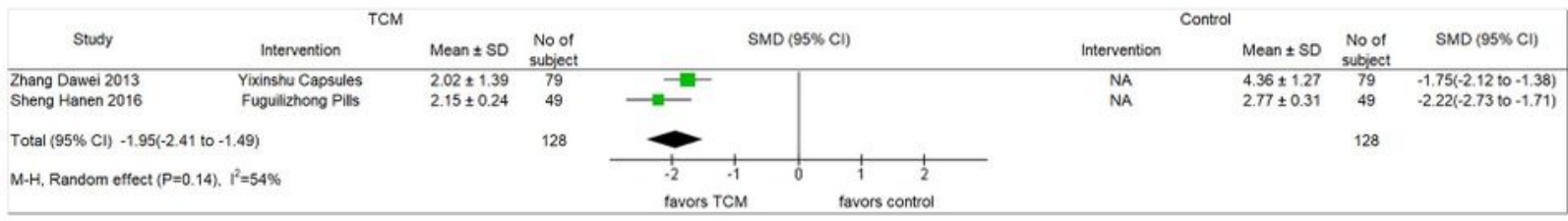

D

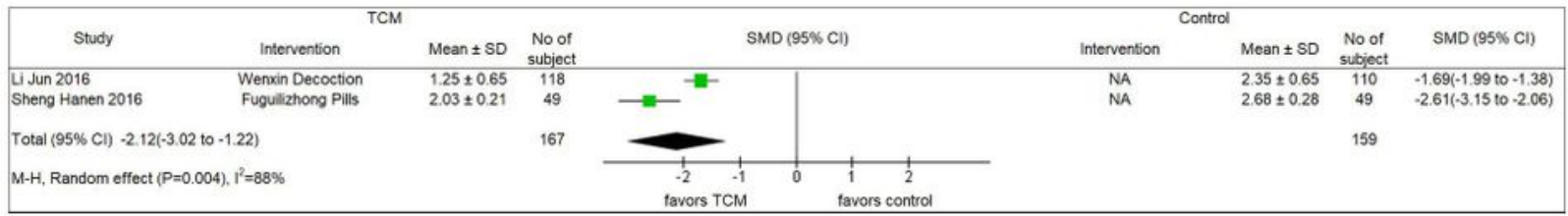

\begin{tabular}{|c|c|c|c|c|c|c|c|c|}
\hline TCM & & & & & & Cont & & \\
\hline Intervention & $\begin{array}{c}\text { No of } \\
\text { eventsitat }\end{array}$ & & & $95 \% \mathrm{Cl})$ & & Intervention & $\begin{array}{c}\text { No of } \\
\text { events }\end{array}$ & $\mathrm{RR}(95 \% \mathrm{Cl})$ \\
\hline Yixinshu Capsules & $70 / 79$ & & & $=$ & & NA & $59 / 79$ & $1.19(1.02$ to 1.38$)$ \\
\hline Quyuxiaoban Capsules & $37 / 41$ & & & $\longrightarrow$ & & Placebo & $31 / 44$ & $1.28(1.03$ to 1.59$)$ \\
\hline Wendan Decoction & $27 / 30$ & & &. & & NA & $23 / 30$ & $1.17(0.93$ to 1.48$)$ \\
\hline Guanmailing Decoction & $28 / 30$ & & & $\longrightarrow$ & & NA & $21 / 30$ & $1.33(1.04$ to 1.72$)$ \\
\hline Maianshu Granules & $27 / 30$ & & & & & NA & $21 / 30$ & $1.29(0.99$ to 1.67$)$ \\
\hline Guanxinkangfu Decoction & $31 / 34$ & & & $\longrightarrow$ & & NA & $21 / 32$ & $1.39(1.06$ to 1.82$)$ \\
\hline 4 to 1.36$)$ & $220 / 244$ & & & & & & $176 / 245$ & \\
\hline$=0.89), P^{2}=0 \%$ & & 0.5 & $\begin{array}{c}0.7 \\
\text { favors control }\end{array}$ & $\begin{array}{c}1.5 \\
\text { favors } \mathrm{TCM}\end{array}$ & $\frac{1}{2}$ & & & \\
\hline
\end{tabular}

\section{Figure 4}

Clinical and angina efficacy comparison between TCM group and control group in UA. A: Clinical efficacy comparison between TCM group and control group. B: Angina efficacy comparison between TCM group and control group. C: Angina frequency comparison between TCM group and control group. D: Nitrates consumption comparison between TCM group and control group. E: ECG comparison between TCM group and control group. 


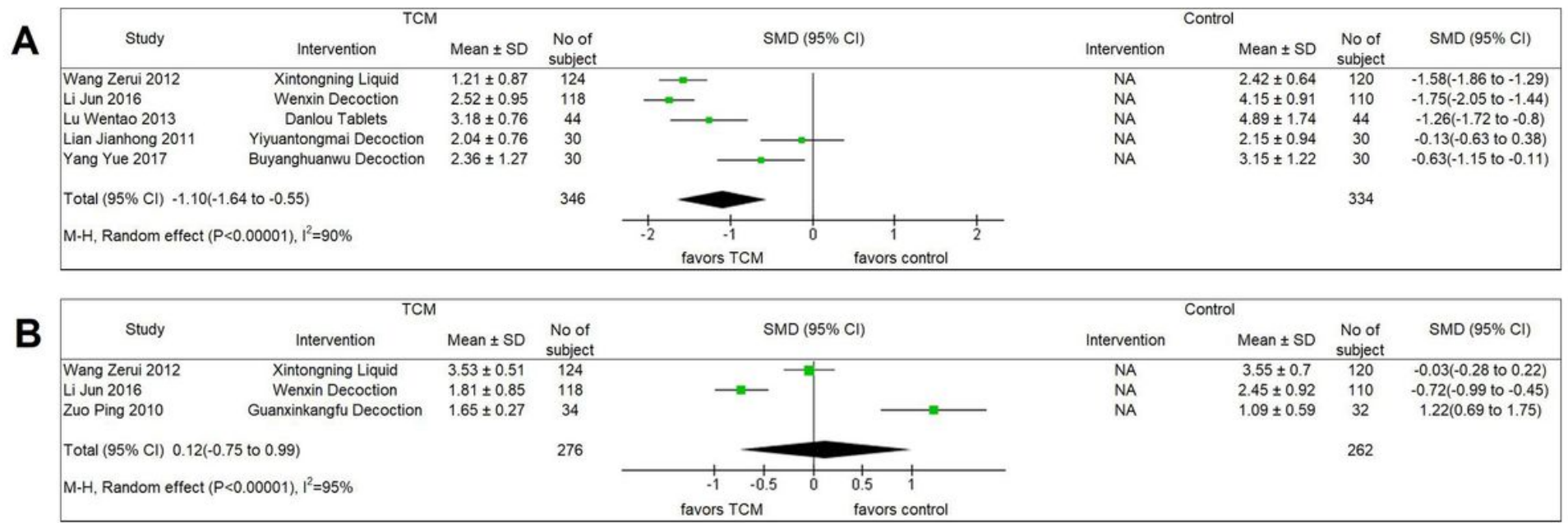

\section{Figure 5}

CRP and LDL-C comparison between TCM group and control group in UA. A: CRP comparison between TCM group and control group. B: LDL-C comparison between TCM group and control group.

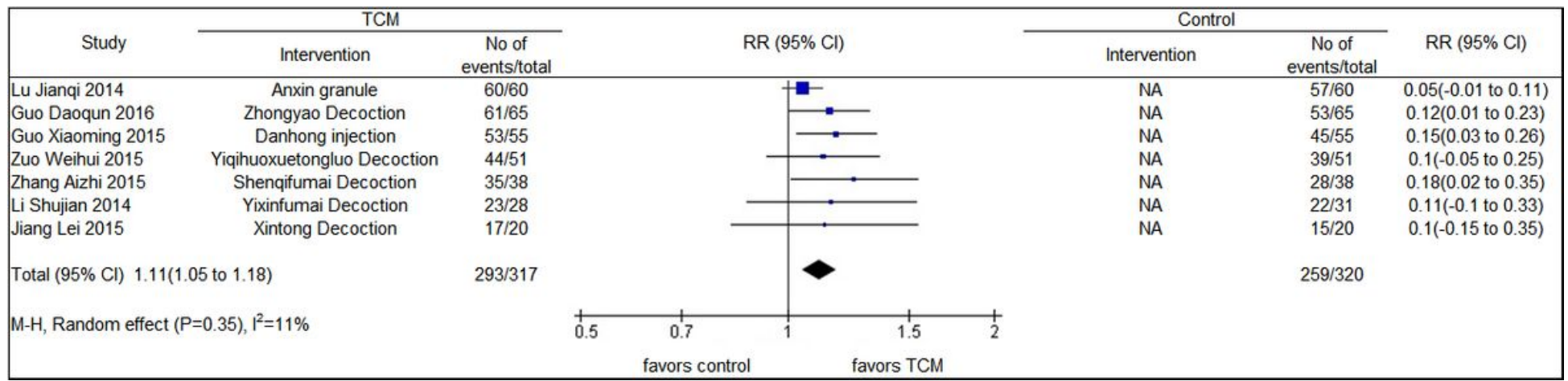

\section{Figure 6}

Clinical efficacy comparison between TCM group and control group in AMI 
A

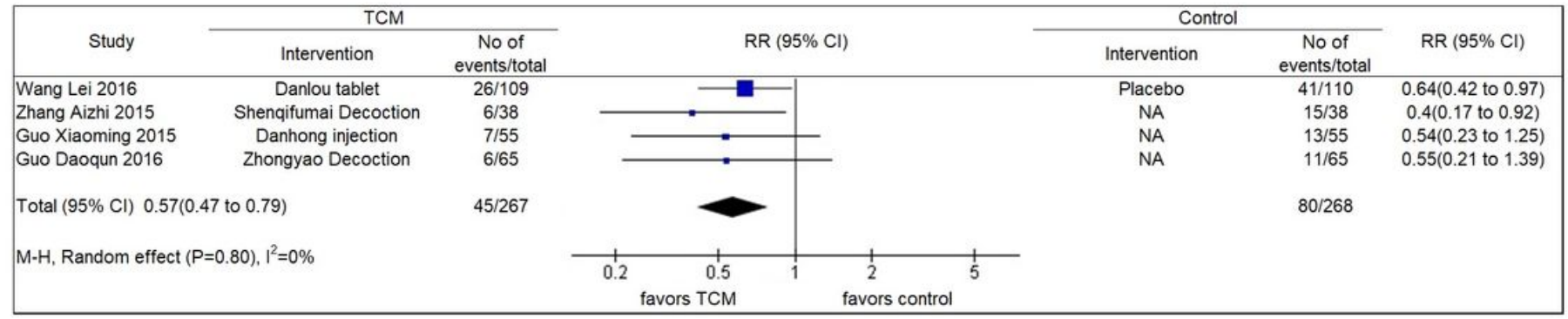

B

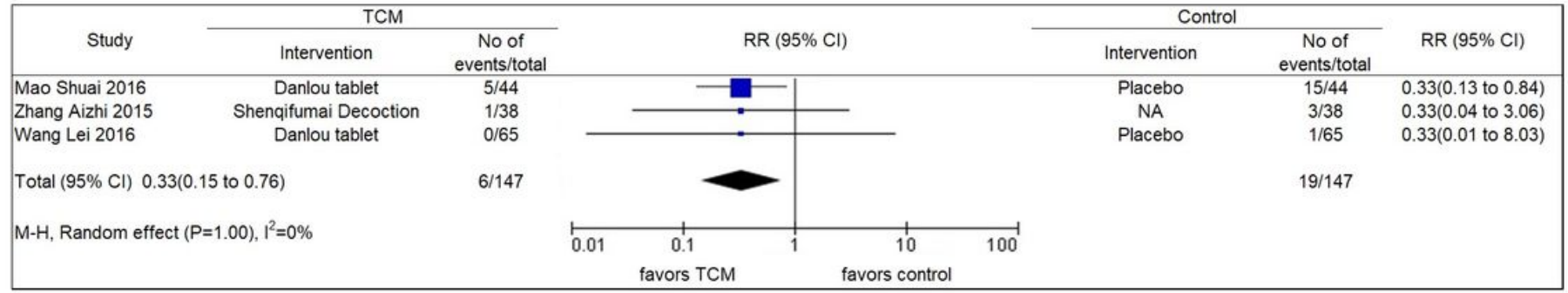

C

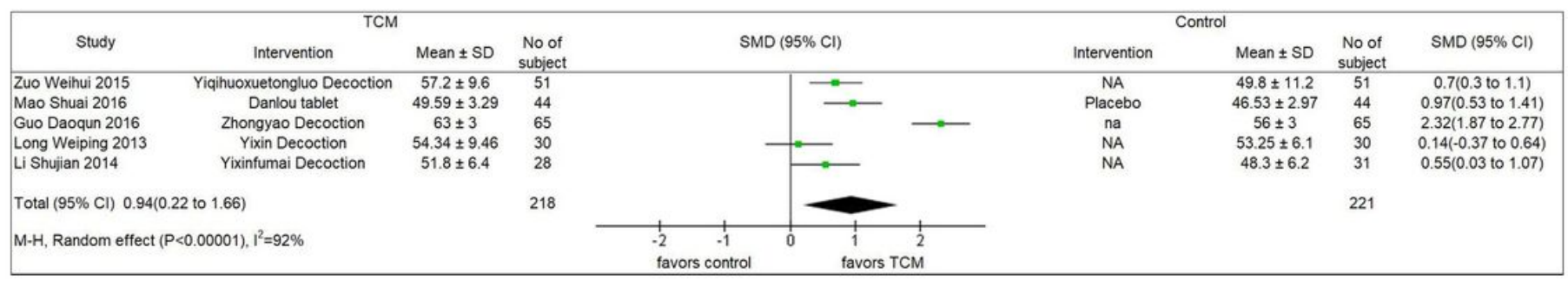

\section{Figure 7}

Major outcomes and LVEF comparison between TCM group and control group in AMI. A: Incidence of MACE comparison between TCM group and control group. B: Incidence of CD comparison between TCM group and control group. C: LVEF comparison between TCM group and control group.

A

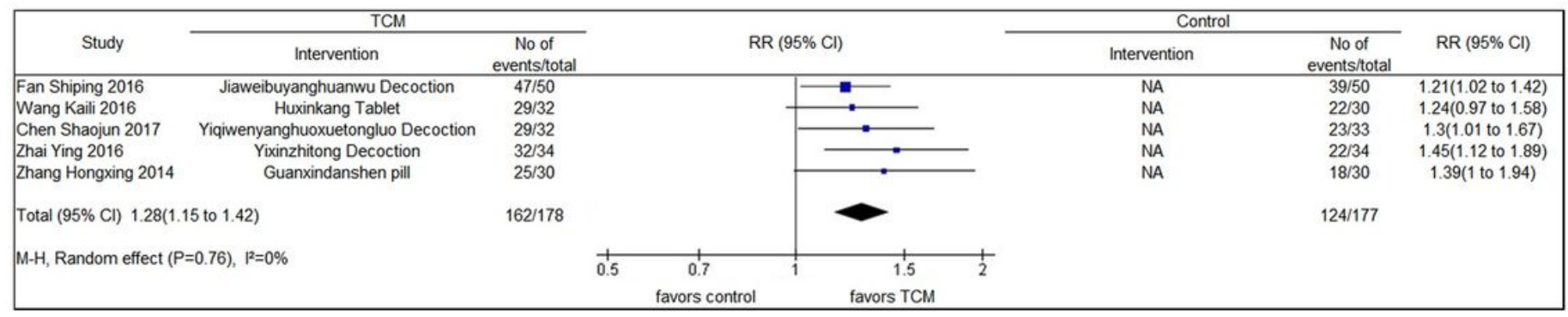

B

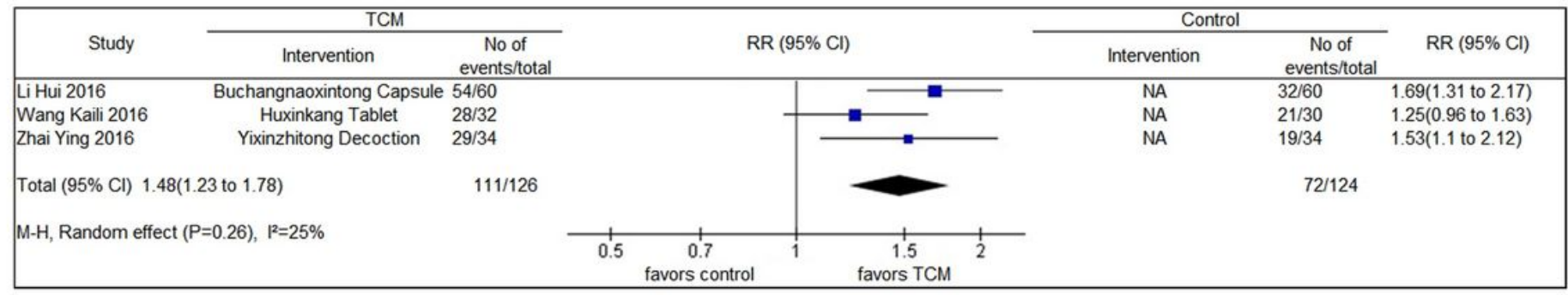

Figure 8 
Clinical and angina efficacy comparison between TCM group and control group in PMI. A: Clinical efficacy comparison between TCM group and control group. B: Angina efficacy comparison between TCM group and control group.

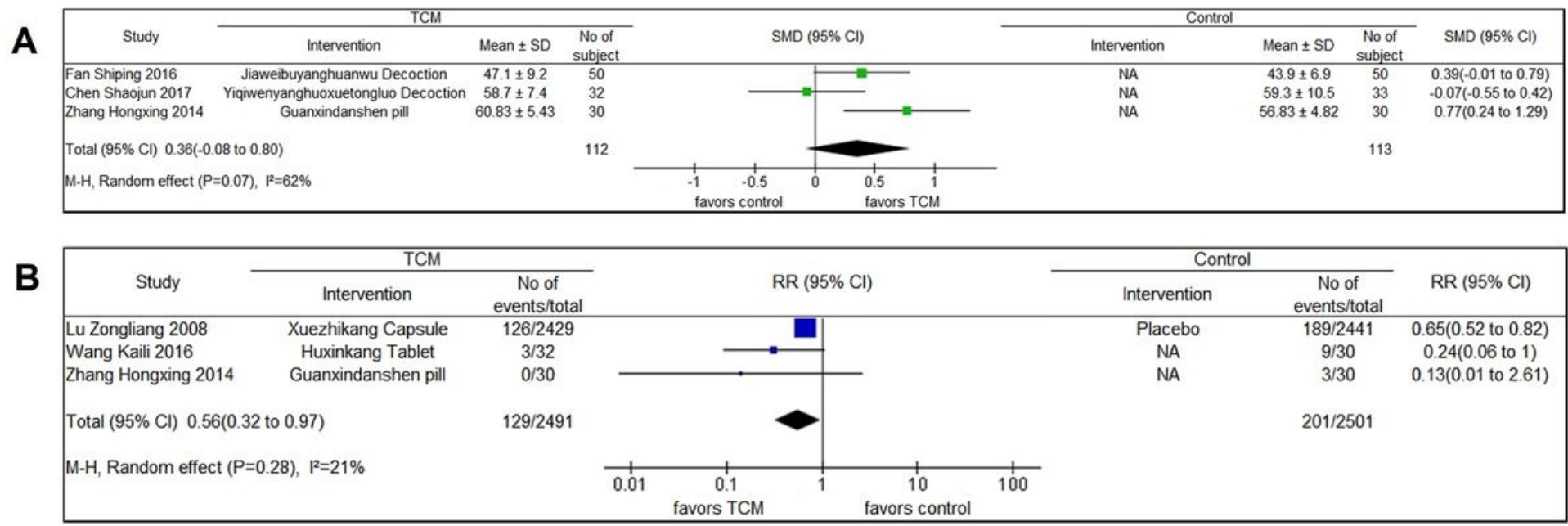

\section{Figure 9}

LVEF and Incidence of MACE comparison between TCM group and control group in PMI. A: LVEF comparison between TCM group and control group. B: Incidence of MACE comparison between TCM group and control group.

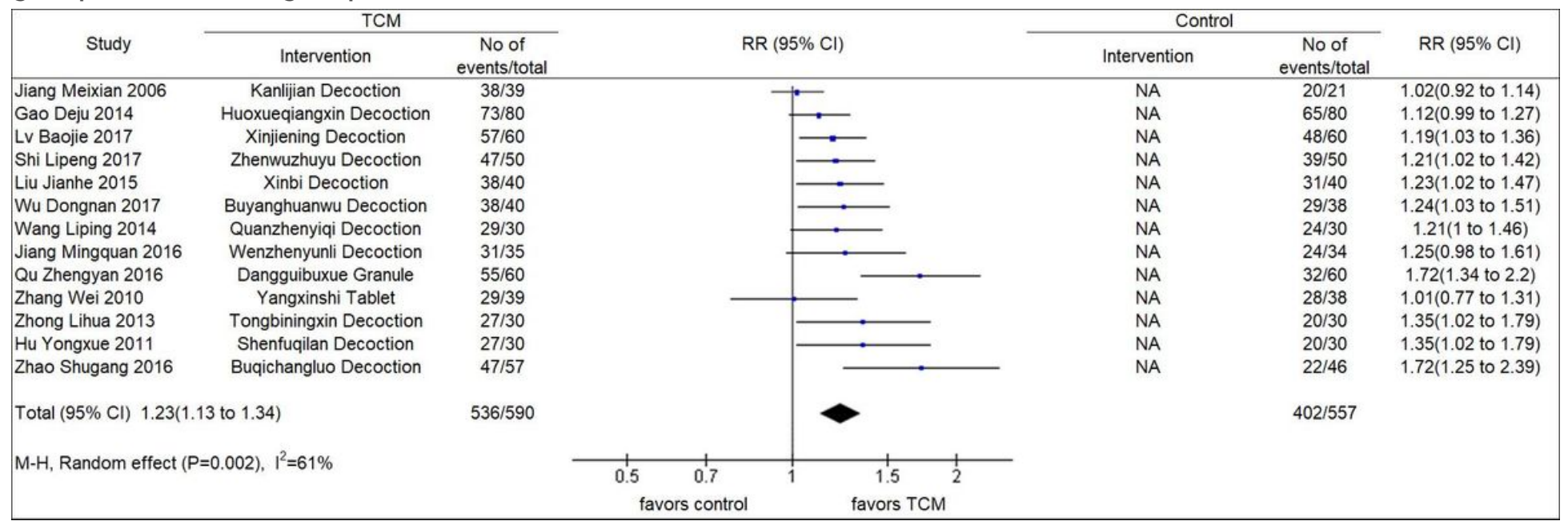

\section{Figure 10}

Clinical efficacy comparison between TCM group and control group in CHF 
A

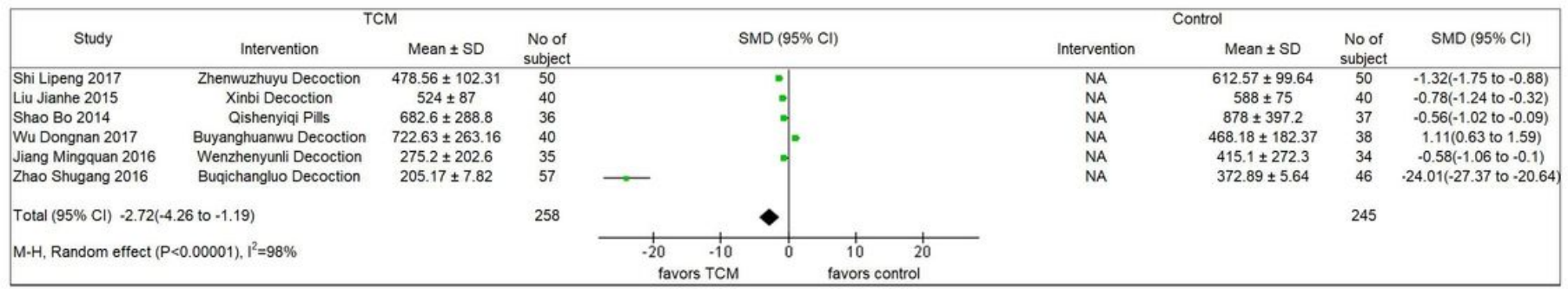

B

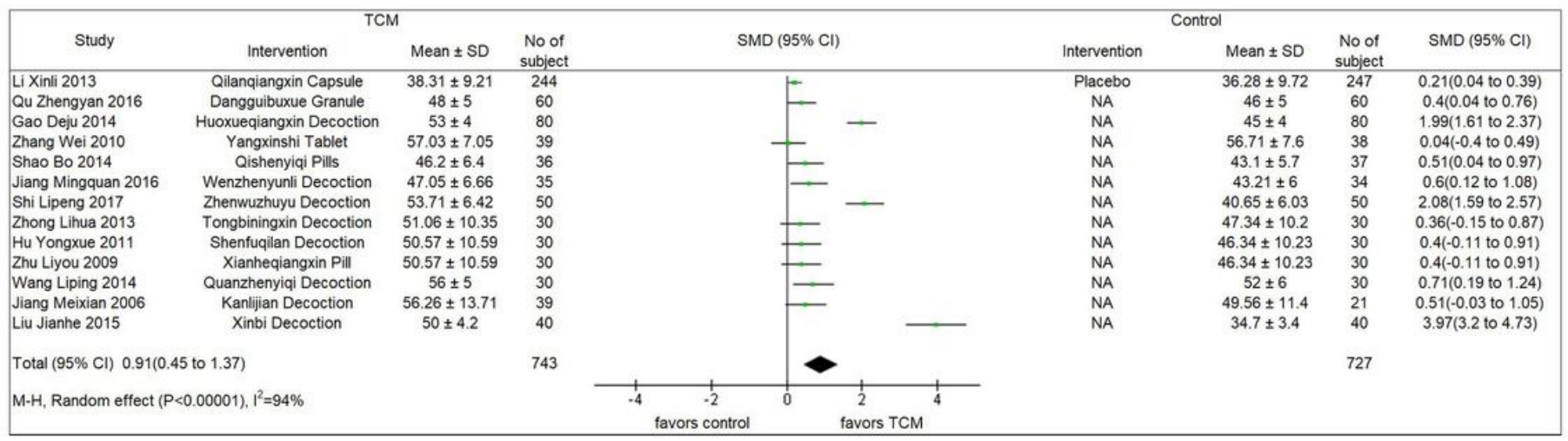

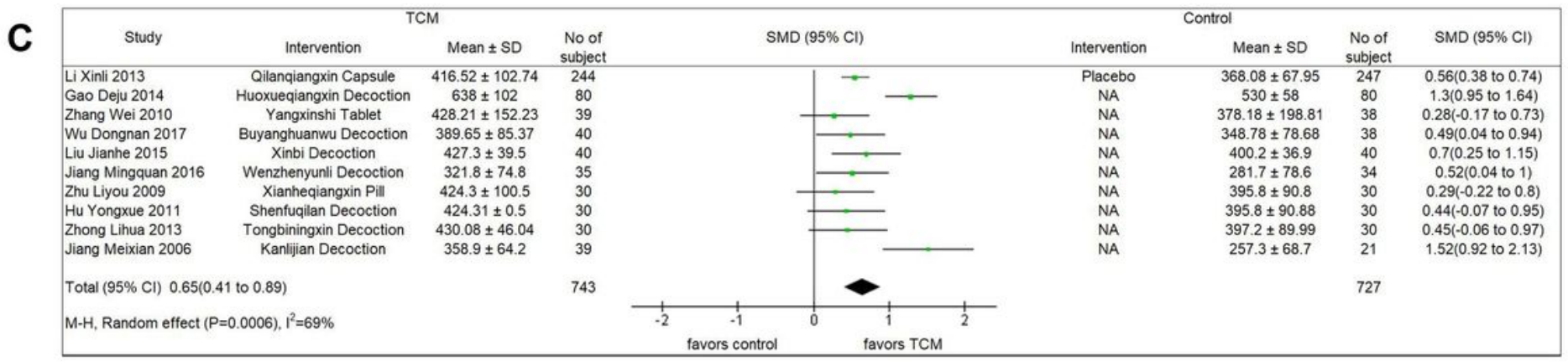

\section{Figure 11}

Comparison of heart failure indicators between TCM group and control group in CHF. A: LVEF comparison between TCM group and control group. B: 6MWT comparison between TCM group and control group in $\mathrm{CHF}$

\section{Supplementary Files}

This is a list of supplementary files associated with this preprint. Click to download.

- table1detailsofincludedstudies.xlsx 